\title{
Inhibition of Human 20S Proteasome by Compounds from Seeds of Psoralea corylifolia
}

\author{
YuJin Kim, Hanna Lee, Euiho Park, and Sang Hee Shim
}

\author{
School of Biotechnologv, Yeungnam Unwersity, Gleongsan 712-749, Korea. E-mail: shshim29ânuackr \\ Recerved June 11, 2009, Accepted July 02, 2009
}

Key Wonds: 20 S proteasome inhibitor. Psoralea corylifolia. Bakuchiol, Isobavachromene. 6-Prenylnaringenin

Psoraleae Semen is the dried seed of Psoralea corylifolia L. (Fabaceae) which is commonly used in traditional Chinese medicine to alleviate asthma and diarrhea and to treat vitiligo and alopecia areata. ${ }^{3}$ Also. this cnide dnig has been used for the treatment of enuresis. pollakiuria. painful feeling of cold in the waist or knees and weak kidney. ${ }^{\text {. }}$ Modem pharmacological and clinical studies have shown that the extracts of $P$. corvifolia possess different biological activities. such as antihyperglycemic. antidepressant, antimicrobial, antitumor and antidernatophytic effects. ${ }^{l}$ The chemical constituents of $P$. corvlifolia include coumarins such as psoralen and isopsoralen. etc. and flavone components such as psoralidin and bavachalcone. etc. ${ }^{2}$

The ubiquitin-proteasome proteolytic ș'stem plays an important role in selective protein degradation and regulates cellular events. including apoptosis. and inflammation. ${ }^{3}$ The $26 \mathrm{~S}$ proteasome is composed of $20 \mathrm{~S}$ core and $19 \mathrm{~S}$ regulator. Since proteasomes interact primarily with endogenous proteins. inhibition of the proteolytic action of the proteasome may block the signaling action of the transcription factor NF- $\mathrm{kB}$ and. thus. inhibit the completion of the cell cycle and hence the mitotic proliferation of cancerous cells, leading to cell death by apoptosis and inhibition of angiogenesis and metastasis. ${ }^{1}$ Cancer cells have been shown to be more sensitive to the proapoptotic effects of proteasome inhibition than normal cells in preclinical evaluation." Thus proteasome inlibitors can be potential anticancer agents.

As part of a project to discover proteasome inhibitory compounds from natural products. several plants were screened using an assay for human $20 \mathrm{~S}$ proteasome inlibition. $\mathrm{A} \mathrm{MeOH}$ extract from Psoraleae Semen with potent 20 S proteasome inhibitory activity was chosen for further chemical investigation. The $\mathrm{MeOH}$ extract of Psoraleae Semen was fractionated depending on the polarity into four fractions. of which each fraction was evaluated on the $20 \mathrm{~S}$ proteasome inhibitory activity. The inhibitory activities of the fractions against $20 \mathrm{~S}$ proteasome were described in a previous report. ${ }^{6}$ Since the $\mathrm{CHCl}_{3}$ fr. (fraction) showed the most potent 20 s proteasome inhibitory activity." the $\mathrm{CHCl}_{3} \mathrm{fr}$. was subjected to chemical investigation for further study. This study reports on the isolation of the compounds from the psoraleae semen and their inhibitory activities on human $20 \mathrm{~S}$ proteasome.

There has been a report that the extract of Psoralea corylifolia inhibited mitochondrial complex I and proteasome activities in SH-SY5Y, a human neuroblastoma cell line, with respect to neurodegenerative diseases such as Parkinson's and Alzheimer's diseases. However. the compounds that are respon- sible for its inhibitory activity have not been identified so far. To identify the active compounds from the extract of Psoraleae Semen, bioassay-guided fractionation was performed using silica gel column chromatography, followed by reversed-phase HPLC. resulting in the isolation of compounds $\mathbf{1 - 8}$. The structures of the isolated compounds were identified as psoralen (1). isopsoralen (2). bakuchiol (3). isobavacluromene (4), 6-prenylnaringenin (5), corylin (6), bavachinin (7), and $د^{3}, 2$-hỵd droxỵbakuchiol (8). The structures of the isolated compounds were identified analyzing their NMR data (Fig. 1) along with comparing their spectral data with those in the literature. ${ }^{28}$ In case of compound 8. initial ${ }^{\mathrm{l}} \mathrm{H}-\mathrm{NMR}$ data was exactly identical to that of $\Delta^{3}, 2$-hydroxybakuchiol. ${ }^{9}$ However 8 easily underwent dehydration to furnish $\Delta^{1,-2}$-bakuchiol (9) of which spectral characteristic was consistent with the structure 9 . $^{\circ}$

To investigate the $20 \mathrm{~S}$ proteasome inlubitory activity of theisolated conpounds from Psoraleae Sement the conunercially available purified human ery throcyte $20 \mathrm{~S}$ proteasome was used. Suc-Leu-Leu-Val-Tyr linked to fluorogenic aninomethylcou-
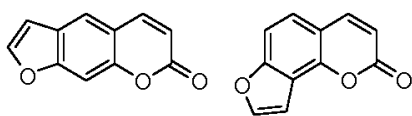

1<smiles>CC1(C)C=[C-]c2c(ccc(C(=O)/C=C/c3ccc(O)cc3)c2O)O1</smiles>

4

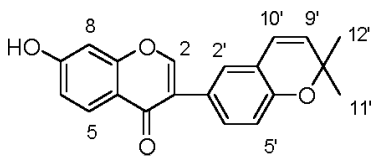

6

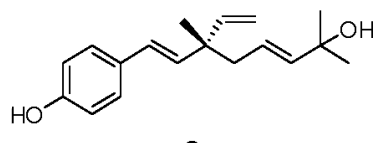

8

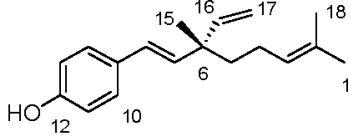<smiles>CC(C)=C[I+]c1c(O)cc2c(c1O)C(=O)CC(c1ccc(O)cc1)O2</smiles>

5<smiles>COc1cc2c(cc1CC=C(C)C)C(=O)CC(c1ccc(O)cc1)O2</smiles>

7

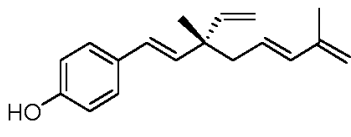

9
Figure 1. Chemical structures of the isolated compounds from Psoraleae Semen. 
Table 1. The inlhibitory activities of the $20 \mathrm{~S}$ proteasome by compounds isolated from Psoralea Semen

\begin{tabular}{cccccccc}
\hline Compounds & Psoralen (1) & Isopsoralen (2) & Bakuchiol (3) & $\begin{array}{c}\text { Isobava } \\
\text { Chromene (4) }\end{array}$ & $\begin{array}{c}\text { 6-Prenyl } \\
\text { naringenin (5) }\end{array}$ & Corylin (6) & Bavachinin (7) \\
\hline $\mathrm{IC}_{50}(\mu \mathrm{M})^{a}$ & $>100$ & $>100$ & $30.5 \pm 1.5$ & $24.3 \pm 1.2$ & $46 \pm 1.2$ & $>100$ & $49 \pm 1.5$ \\
\hline
\end{tabular}

${ }^{2} \mathrm{IC}_{50}\left(50^{\circ}{ }^{\circ} .0\right.$ inhibition concentrations) were calculated from a log dose inhibition curve and expressed as the mean \pm SD of triplicate experiments. Epoximicin was used as the positive control ( $\mathrm{IC}_{50}=65 \mathrm{nM}$ ).

marin (Suc-LLVY-AMC) was used as a substrate to determine the chymotrypsin-like activity of $20 \mathrm{~S}$ proteasome. The inhibitory activity was detennined by measuring the generation of free AMC using a fluorescence plate reader after adding SucLLVY-AMC to incubated mixtures of the isolated compounds and $20 \mathrm{~S}$ proteasome. The inhibitory activities of the isolated compounds against $20 \mathrm{~S}$ proteasome are shown in Table 1 . Compounds 3.4 .5 and 7 inlibited the activity of $20 \mathrm{~S}$ proteasome in a dose-dependent manner with $\mathrm{IC}_{50}$ values of $30.5 \pm 1.5$. $24.3 \pm 1.2 .46 \pm 1.2$. and $49 \pm 1.5 \mu \mathrm{M}$. respectively. Compound 8 was not evaluated using this assay since $\mathbf{8}$ was easily dehydrated to 9. A known proteasome inhibitor, epoximicin (IC $\mathrm{IC}_{\mathrm{s})}=$ $65 \mathrm{nM}$ ). was employed as a positive control in the assay

Since the first proteasome inhibitor. Bortezomib (Velcade ${ }^{\mathrm{TM}}$. Millenium) was approved by the US FDA for the treatment of multiple myeloma in $2003,{ }^{10}$ natural products as well as synthetics have been vigorously investigated for working as proteasome inhibitors and antitumor agents. Several proteasome inhibitors such as NPI- $00522^{11}$ lactacystin. ${ }^{12}$ withaferin $\mathrm{A}^{13}$ celastrol. ${ }^{14}$ gliotoxin. ${ }^{15}$ ginsenoside Rd. ${ }^{16}$ etc were already identified from natural sources including microsomes. Yang et al. reported the relationship between proteasomal chymoty.psin-like activity and tumor growth, demonstrating that treatment of withaferin $\mathrm{A}$ resulted in inhibition of tumor growth in association with inhibition of the tumor tissue proteasomal chy'motrypsin-like activity. $^{13}$

Several biological studies of Psoraleae Semen have been performed. ${ }^{17}$ As mentioned in the above, there has been a report that the extract of Psoraleae Semen inhibited mitochondrial complex I and proteasome activities in $\mathrm{SH}-\mathrm{SY} 5 \mathrm{Y}$. assuming that the interplay of oxidative damage due to reactive species generation, mitochondrial complex I inhibition. and proteasomal dysfunction may be responsible for the reduced cell viability in SH-SY5Y neuroblastoma cells treated with Psoraleae Semen. possibly leading to increase in the risk of development of neurodegenerative diseases such as Parkinson's and Alzheimer's diseases. However. even though the inhibitory activity' of $P$. corviffolia on proteasome has been reported before, the compounds from the extract that may be responsible for the activity were identified in this study for the first time. Moreover, 20 s proteasome purified from human erythrocyte was used to determine the chymotry psin-like activity of proteasome in this study whereas proteasome-containing SH-SY5Y cells which were ly'sed with cell ly sis buffer were used without purification in the above mentioned report.

In conclusion our data show that Psoraleae Semen may exhibit the antitumor activity by inhibiting human $20 \mathrm{~S}$ proteasome and bakuchiol (3), isobavachromene (4). 6-prenylnaringenin (5). and bavachinin (7) from this plant can contribute to the inhibitory activity on human $20 \mathrm{~S}$ proteasome.

\section{Experimental Section}

Plant mateial. The seeds of $P$. corvilfoli $a$ were purchased from the Daegu pharmacopoeia market in South Korea. A voucher specimen has been deposited in Natural Products Chemistry Laboratory of School of Biotechnology. Yeungnam University

Reagents and instruments. NMR spectra were recorded with Varian's standard pulse program of Varian VNS spectrometer at $250 \mathrm{MHz}$. $300 \mathrm{MHz}$. and $600 \mathrm{MHz}$. EI-MS spectra were recorded with Micromass spectnum (AUTOSPEC. UK). TLC was done using Kieselgel 60F254 (Merck) and RP-18 (Whatman). Column chromatography was done using silica gel (70-230 mesh. Merck). Agilent 1200 series HPLC system equipped with a quaternary punp, a degasser. an injector, a column thermostat. and diode array detector (DAD) was used for purification of compounds. All HPLC separations were carried out using an Eclipse XDB- $C_{18}$ semi-preparative column $(9.4 \times 250 \mathrm{~mm} .5$ $\mu \mathrm{m}$. Agilent Technologies. USA) at a flow rate of $2 \mathrm{~mL} / \mathrm{min}$. A commercially available 20 S Proteasone Assay Kit (AK-740. BIOMOL Intemational. LP.) was used. which provided the purified. human erythrocyte $20 \mathrm{~S}$ proteasome, assay buffer. and substrate Suc-LLVY-AMC. Proteasome inhibition was determined using a fluorescence plate reader (FluorOptima. BMG Labtech Ltd., UK) to measure fluorescence of free AMC from Suc-LLVY-AMC.

Extraction, fractionation and isolation. The dried Psoraleae Semen $(\mathrm{l} \mathrm{kg})$ was extracted with $\mathrm{MeOH}$ and the extracts were successively partitioned with $n$-hexane, $\mathrm{CHCl}_{3}$, EtOAc. and $n-\mathrm{BuOH}$. The $\mathrm{CHCl}_{3}$ fraction $(96.5 \mathrm{~g}$ ) with potent 20 S proteasome inhibition activity ${ }^{6}$ was subjected to silica gel column chromatograply eluting with $n$-hexane and EtOAc gradient $\operatorname{sistem}(10: 1 \rightarrow 10: 4)$, affording twenty'-seven fractions (Fr. I Fr. 27). Fraction 3 gave a colorless oil. compound 3 . Fraction 13 and 15 were crystallized from $n$-hexane. EtOAc. and $\mathrm{MeOH}$ to yield compounds 1 and 2 . respectively. Fraction 16 was subjected to repeated column chromatography with a gradient elution of $n$-hexane/EtOAc $(10: 1 \rightarrow 10: 1.5)$. resulting in 19 subfractions (Fr. 16-1 $\sim$ Fr. 16-19). Compound 4 was isolated through repeated column chromatography eluted with Hexane and $\mathrm{CH}_{2} \mathrm{Cl}_{2}$ gradient solvents followed by reversed-phase HPLC (30 to $100 \% \mathrm{CH}_{3} \mathrm{CN}$ in $\mathrm{H}_{2} \mathrm{O}$ over $40 \mathrm{~min}$ ). Fraction 24 of the $\mathrm{CHCl}_{3}$ fraction was chromatographed on a silica gel column with a gradient elution of $n$-hexane and EtOAc $(10: 1 \rightarrow 1: 1)$ to afford fifty subfractions (Fr. 24-I $\sim \mathrm{Fr}$. 24-50). Fr. 24-7 and Fr. 24-20 were subjected to semi-preparative HPLC (20 to $100 \% \mathrm{CH}_{3} \mathrm{CN}$ in $\mathrm{H}_{2} \mathrm{O}$ over $40 \mathrm{~min}$ ) to afford compounds 5 and 6. respectively. Conpounds 7 and 8 were purified from $\mathrm{Fr}$. $24-26$ and $\mathrm{Fr}$. 24-30, respectively through semi-preparative HPLC ( 10 to $100 \% \mathrm{CH}_{2} \mathrm{CN}$ in $\mathrm{H}_{2} \mathrm{O}$ over 45 min).

Psoralen (1). Colorless cry stals. ${ }^{-} \mathrm{H}-\mathrm{NMR}\left(250 \mathrm{MHz} . \mathrm{CDCl}_{\mathfrak{s}}\right)$ 
ò 7.78 (1H. d. $J=9.6 \mathrm{~Hz} . \mathrm{H}-4) .7 .68\left(1 \mathrm{H}, \mathrm{d}, J=2.4 \mathrm{~Hz}, \mathrm{H}-2^{\prime}\right)$, 7.67 (lH. s. H-5). $7.46(1 \mathrm{H}$. br s. H-8) $6.82(\mathrm{lH} . \mathrm{dd} J=0.9 .2 .4$ $\mathrm{Hz}, \mathrm{H}-3) .6 .36$ (1H. d. $J=9.6 \mathrm{~Hz} . \mathrm{H}-3):{ }^{13} \mathrm{C}-\mathrm{NMR}(62.5 \mathrm{MHz}$. $\left.\mathrm{CDCl}_{3}\right)$ ò $161.10(\mathrm{C}-2), 156.45(\mathrm{C}-7), 152.09(\mathrm{C}-9), 146.93$ $\left(\mathrm{C}-2^{\prime}\right) .14+06(\mathrm{C}-4) .124 .89(\mathrm{C}-6) .119 .85(\mathrm{C}-5) .114 .70(\mathrm{C}-3)$. $106.39\left(\mathrm{C}-3^{\prime}\right) .99 .91(\mathrm{C}-8)$ : EIMS $m z$ (rel. int.) $186[\mathrm{M}]^{+}(98)$. $158\left[\mathrm{M}-\mathrm{CO}^{+}(100)\right.$.

Isopsoralen (2). Colorless crystals. ${ }^{1} \mathrm{H}-\mathrm{NMR}$ (250 MHz. $\mathrm{CDCl}_{3}$ ) $\delta 7.78$ (1H. d. $J=9.6 \mathrm{~Hz} . \mathrm{H}-4$ ). 7.66 (1H. br d. $J=2.1$ $\left.\mathrm{Hz}, \mathrm{H}-2^{\prime}\right) .7 .40$ (lH, d $\left.J=8.5 \mathrm{~Hz}, \mathrm{H}-6\right) .7 .3+(1 \mathrm{H}, \mathrm{d} . J=8.5 \mathrm{~Hz}$. H-5). 7.09 (1H. m, H-3'), 6.36 (1H, d. $J=9.6 \mathrm{~Hz} . \mathrm{H}-3)$ : ${ }^{13} \mathrm{C}-\mathrm{NMR}\left(62.5 \mathrm{MHz}, \mathrm{CDCl}_{3}\right)$ o $160.8 \mathrm{l}(\mathrm{C}-2) .157 .35(\mathrm{C}-7)$. 148.50 (C-9). 145.88 (C-2'), 144.50 (C-4). 123.83 (C-5). 116.92 (C-8). $11+12$ (C-3). 113.52 (C-10). 108.80 (C-6). 104.09 (C-3'): EIMS $m z$ (rel. int.) $186\left[\mathrm{M}^{-}(100) .158\left[\mathrm{M}-\mathrm{CO}^{-}(85)\right.\right.$.

Baluchiol (3). Colorless oil. ${ }^{\mathrm{H}} \mathrm{H}-\mathrm{NMR}\left(250 \mathrm{MHz} . \mathrm{CDCl}_{3}\right)$ ò $7.14(2 \mathrm{H}, \mathrm{d}, J=8.4 \mathrm{~Hz}, \mathrm{H}-10.14) .6 .68(2 \mathrm{H}, \mathrm{d} . J=8.4 \mathrm{~Hz}$. $\mathrm{H}-11,13) .6 .18(1 \mathrm{H}, \mathrm{d} . J=16 \mathrm{~Hz} . \mathrm{H}-8), 5.98(1 \mathrm{H}, \mathrm{d} . J=16 \mathrm{~Hz}$, $\mathrm{H}-7) .5 .79(\mathrm{lH}, \mathrm{dd} . J=10.17 \mathrm{~Hz}, \mathrm{H}-16), 5.04(1 \mathrm{H}$, br t. H-3). $4.93(2 \mathrm{H}, \mathrm{m}, \mathrm{H}-17) .1 .89(2 \mathrm{H}, \mathrm{m}, \mathrm{H}-5), 1.59(3 \mathrm{H} . \mathrm{s} . \mathrm{H}-18), 1.50$ (3H, s, H-1) 1.41 (2H. s. H-4). $1.11(3 \mathrm{H}, \mathrm{s}, \mathrm{H}-15) ;{ }^{13} \mathrm{C}-\mathrm{NMR}(62.5$ $\left.\mathrm{MHz} . \mathrm{CDCl}_{3}\right)$ ò 131.1 (C-9). $127.3(\mathrm{C}-10) .115 .4(\mathrm{C}-1 \mathrm{l}) .154 .2$ (C-12). 115.4 (C-13), 127.3 (C-14), 135.8(C-8), 126.4 (C-7), 42.4 $(\mathrm{C}-6),+1.2(\mathrm{C}-5), 23.2(\mathrm{C}-4), 12+.8(\mathrm{C}-3), 131.2(\mathrm{C}-2) .17 .6$ (C-1), $25.6(\mathrm{C}-18) .23 .2(\mathrm{C}-15), 145.8(\mathrm{C}-16) .111 .9(\mathrm{C}-17)$.

Isobavachromene (4). Yellow amorphous powder. ${ }^{3} \mathrm{H}-\mathrm{NMR}$ $\left(600 \mathrm{MHz}, \mathrm{CDCl}_{3}\right)$ ò $7.82(1 \mathrm{H}, \mathrm{d}, J=16 \mathrm{~Hz}, \mathrm{H}-\beta) .7 .69(1 \mathrm{H}$. d. $\left.J=9.0 \mathrm{~Hz}, \mathrm{H}-6^{\prime}\right) .7 .55(2 \mathrm{H}, \mathrm{d}, J=8.4 \mathrm{~Hz} . \mathrm{H}-2.6), 7.42(1 \mathrm{H}$, d. $J=16 \mathrm{~Hz}, \mathrm{H}-\alpha), 6.86(2 \mathrm{H}, \mathrm{d} . J=8.4 \mathrm{~Hz}, \mathrm{H}-3.5), 6.7+(\mathrm{lH}$. d. $\left.\left.J=10 \mathrm{~Hz} . \mathrm{H}-\mathrm{l}^{\prime \prime}\right) .6 .36(1 \mathrm{H}, \mathrm{d}, J=9.0 \mathrm{~Hz}, \mathrm{H}-5)^{\prime}\right) .5 .57(\mathrm{lH}$, d. $\left.J=10 \mathrm{~Hz}, \mathrm{H}-2^{\prime \prime}\right), 1.45\left(6 \mathrm{H}, \mathrm{s} . \mathrm{H}_{3}-4^{\prime \prime} .5 "\right)$.

6-Prenylnaringenin (5). Yellow amorphous powder. ${ }^{1} \mathrm{H}-\mathrm{NMR}$ (300 MHz, DMSO- $\left.d_{6}\right)$ ò 12.41 (1H. s, 5-OH). $7.30(2 \mathrm{H}$, br d. $\left.J=8 .+\mathrm{Hz}, \mathrm{H}-2^{\prime}, 6^{\prime}\right), 6.79\left(2 \mathrm{H}\right.$, br d $\left.. J=8.4 \mathrm{~Hz} . \mathrm{H}-3^{\prime}, 5^{\prime}\right), 5.96$ (1H. s, H-8). 5.40 (1H. dd. $J=3.13$ Hz. H-2). 5.12 (1H. br t, $\left.J=6.9 \mathrm{~Hz}, \mathrm{H}-2^{\prime \prime}\right) .3 .25\left(2 \mathrm{H}\right.$. br d $\left.J=6.9 \mathrm{~Hz}, \mathrm{H}_{\alpha}-\mathrm{I}^{\prime \prime}, \mathrm{H}_{\mathrm{\beta}}-\mathrm{l}^{\prime \prime}\right)$. $3.17\left(1 \mathrm{H}\right.$, dd $\left.. J=13.17 \mathrm{~Hz} . \mathrm{H}_{\beta}=3\right), 2.67(1 \mathrm{H}, \mathrm{dd} . J=3,17 \mathrm{~Hz}$, $\left.\mathrm{H}_{1}-3\right) .1 .69\left(3 \mathrm{H}, \mathrm{s} . \mathrm{H}_{3}-5^{\prime \prime}\right) .1 .6 \mathrm{I}$ (3H. s. $\mathrm{H}_{3}-4 "$ ).

Corylin (6). Yellow cristals. ${ }^{1} \mathrm{H}-\mathrm{NMR}\left(300 \mathrm{MHz}\right.$. DMSO- $t_{6}$ ) o 8.33 (1H. s. H-2), 7.96 (1H, d. $J=8.7 \mathrm{~Hz}, \mathrm{H}-5) .7 .30$ (1H. dd, $\left.J=2.4 .8 .1 \mathrm{~Hz} . \mathrm{H}-6^{\prime}\right) .7 .29$ (1H. br s. H-2') $6.90(1 \mathrm{H}, \mathrm{dd} . J=$ $2.1,8.7 \mathrm{~Hz}, \mathrm{H}-6), 6.85(1 \mathrm{H} . \mathrm{d} . J=2.1 \mathrm{~Hz} . \mathrm{H}-8) .6 .78(1 \mathrm{H}, \mathrm{d}$, $J=8.1 \mathrm{~Hz}, \mathrm{H}-5), 6.43\left(1 \mathrm{H}, \mathrm{d}, J=9.9 \mathrm{~Hz}, \mathrm{H}-10^{\prime}\right), 5.78(1 \mathrm{H}, \mathrm{d}$. $\left.J=9.9 \mathrm{~Hz} . \mathrm{H}-9^{\prime}\right) .1 .40\left(6 \mathrm{H} . \mathrm{s}, 2 \times \mathrm{CH}_{3}\right) .{ }^{13} \mathrm{C}-\mathrm{NMR}(75 \mathrm{MHz}$. DMSO-d $\left.d_{6}\right) \delta 175.0(\mathrm{C}-4) .163 .2(\mathrm{C}-7), 157.9(\mathrm{C}-9), 153.6$. 152.6 (C-4'. C-2) 131.7. 130.1 (C-2', C-6'). 127.7(C-5). 127.4. $12+.9\left(\mathrm{C}-9^{\prime} . \mathrm{C}-10^{\prime}\right), 123.6\left(\mathrm{C}-1^{\prime}\right), 122.2\left(\mathrm{C}-5^{\prime}\right), 121.0(\mathrm{C}-3)$. $116.9\left(\mathrm{C}-3^{\prime}\right), 116.0(\mathrm{C}-10) .115 .7(\mathrm{C}-6) .102 .6(\mathrm{C}-8) .76 .7(\mathrm{C}-8)$. 49.0. $28.2\left(\mathrm{C}-11^{\prime}\right.$ or $\left.\mathrm{C}-12^{\prime}\right)$

Bavachinin (7). Yellow amorphous powder. ${ }^{~ H}-\mathrm{NMR}(300$ $\left.\mathrm{MHz} . \mathrm{CDCl}_{3}\right)$ ò 7.65 (1H. s. H-5). $7.33(2 \mathrm{H}, \mathrm{d} J=8.4 \mathrm{~Hz}, \mathrm{H}-2$ '. $\left.6^{\prime}\right), 6.86\left(2 \mathrm{H}, \mathrm{d}, J=8.4 \mathrm{~Hz}, \mathrm{H}-3^{\prime}, 5^{\prime}\right) .6 .42(\mathrm{HH}, \mathrm{s}, \mathrm{H}-8) .5 .36(\mathrm{lH}$. dd $J=3.0,13 \mathrm{~Hz}, \mathrm{H}-2), 5.25\left(\mathrm{lH}, \mathrm{t} . J=6.6 \mathrm{~Hz}, \mathrm{H}-2^{\prime \prime}\right) .3 .82(3 \mathrm{H}$. 5, 7-OMe). $3.22\left(2 \mathrm{H}, \mathrm{d}, J=6.6 \mathrm{~Hz} . \mathrm{H}_{2}-\mathrm{l}^{\prime \prime}\right) .3 .00$ (1H, dd. $J=$ $\left.13.17 \mathrm{~Hz}, \mathrm{H}_{\beta}-3\right) .2 .74\left(\mathrm{lH} . \mathrm{dd} J=3.0 .17 \mathrm{~Hz}, \mathrm{H}_{0}-3\right), 1.71(3 \mathrm{H}$. 5. $\left.\mathrm{H}_{3}-4^{\prime \prime}\right), 1.67$ (3H, s. $\left.\mathrm{H}_{3}-5^{\prime \prime}\right)$.

$\Delta^{\mathbf{3}}$,2-Hydroxy baluchiol (8). Oil. ${ }^{\mathrm{H}} \mathrm{H}-\mathrm{NMR}\left(300 \mathrm{MHz}, \mathrm{CDCl}_{3}\right)$ ò $7.2 \mathrm{l}(2 \mathrm{H}, \mathrm{d} . J=8.4 \mathrm{~Hz} . \mathrm{H}-10.14) .6 .7+(2 \mathrm{H}, \mathrm{d}, J=8.4 \mathrm{~Hz}$.
$\mathrm{H}-11.13) .6 .23(1 \mathrm{H} \mathrm{d} . J=17 \mathrm{~Hz} \mathrm{H}-8) .6 .03(1 \mathrm{H} \mathrm{d} . J=17 \mathrm{~Hz} \mathrm{H}-7)$, $5.86(\mathrm{lH}, \mathrm{dd}, J=1 \mathrm{l}, 17 \mathrm{~Hz}, \mathrm{H}-16), 5.60(2 \mathrm{H}, \mathrm{m}, \mathrm{H}-3,4) .5 .02$ (lH, d, $J=11 \mathrm{~Hz}, \mathrm{H}-\mathrm{I} 7 \mathrm{~b}$ ). 4.99 (lH. d, $J=17 \mathrm{~Hz}, \mathrm{H}-\mathrm{I} 7 \mathrm{a}$ ). 2.20 (2H. d. $J=6$ Hz. H-5). 1.28 (6H. s. H-1, 18), 1.15 (1H, s, H-15).

Proteasome inhibition assay. The inhibition assay was done as follows. ${ }^{6} 0.03 \%$ sodium dodecyl sulfate was added to assay buffer to activate the $20 \mathrm{~S}$ proteasome s chymotrypsin-like activity. The assay buffer was added to the blank and sample plate, and a diluted solution of positive control was added to the inhibitor wells. The enriched proteasome fraction was diluted to a final assay concentration of $50 \mu \mathrm{g} / \mathrm{mL}$ using assay buffer. This diluted fraction was then added to each well. and then. the plate was preincubated for $10 \mathrm{~min}$ at $37^{\circ} \mathrm{C}$ to allow the inhibitor and enzyme to interact. The enzymatic reaction was started by adding Suc-LLVY-AMC substrate to a final concentration of $10 \mu \mathrm{M}$. The chy motrypsin-like enzy matic activity of the proteasome was determined by measuring the generation of free AMC using a fluorescent plate reader (FluorOptinta, BMG LabTech Ltd. UK) capable of excitation at a wavelength of $355 \mathrm{~nm}$ and detection of enitted light at $460 \mathrm{~nm}$. Epoximicin was used as the positive control $\left(\mathrm{IC}_{50} 65 \mathrm{nM}\right)$ which is a rapid. potent and irreversible inhibitor of the $20 \mathrm{~S}$ proteasome chymotrypsin-like activity. It can also inhibits the proteasone's trypsinlike and peptidyl-glutamyl peptide hy̆drolase activities. but at 100 - and 1.000 -fold slower rate. respectively.

Acknowledgments. This research was supported by the Korea Research Foundation grant (KRF-2007-531-E00113)

\section{References}

1. Qiao, C.-F.; Han, Q.-B.; Song, J.Z.; Mo, S.-F : Xu, H.-X. J. Sep. Sci. $2007,30,813$

2. Zhao, L.; Huang, C.; Shan, Z.; Xiang, B.; Mei, L. J. Chrontatogr. $B 2005,821,67$

3. (a) Glickman, M. H. Maxtal, V. Cun Top. Aicrobiol Immunol. 2002, 268, 43. (b) Hershko, A.; Ciechanover, A. Amm. Rev' Biochent 1998,67, 425

4. Almond, J. B.: Cohen, G. M. Lethemia 2002, 16, 433.

5. Adams, T. Nat Rev $2004,4,349$

6. (a) Shim, S. H. Kor J Phamacogn $2008,39,56$ (b) Shim, S H.: Balek K.-H.: Kim Y. S. Bull Korean Chem Soc. 2009, 30, 1385

7. Tang, S. Y.; Gruber, J.; Wong, K. P.; Halliwell, B. Ann. X. Y. Acad. Sci. 2007, 1100, 486

8. Guo J.; Weng, X.; Wu, H.; Li, Q.; Bi, K. Food Chemistry 2005, 91.287.

9. Shah, C. C. Bhalla, V. K.; Dev, S. J. Indion Chem. Soc: 1997, 7f, 970. 10. Paramore. A.: Frantz, S. Kat. Rer. 2003, 2.611

11. (a) Ruiz, S.; Krupnik, Y.; Keating, M.; Chandra, J.; Palladino, M.; McConkey, D. Aol Cancer Ther 2016, 5, 1836 (b) Groettrup, M.: Schmidthe, G. Drug Discov. Today 1999, 4,63

12. Omura, S.: Matsuzaki, K.: Fujimoto, T: Kosuge, K.: Fuzuya, T.; Fujita, S.: Nakagawa, A. J. Antibiot. 1991, ff, 113

13. Yang, H.: Shi, G.; Dou, Q. P. M fol Phamacol 2007, 71, 426.

14. Yang. H.; Chen, D.; Cui, Q. C.; Yuan, X.; Dou, Q. P. Cancer Res. 2006, 66,4758

15. Andre P.: Claudine C.: Jeant, D.: Paule, R Pansitol. Res. 2002, 88, 785. 16. Chang. T.: Ding. H.: Kè, Y. J. Agric. Food Chem. 2008, 56, 12011

17. (a) Latha, P. G.: Evans, D. A.: Panikkar, K. R.: Tavavardhanan, K. K. Fitoterapia 2000, 71, 223. (b) Latha. P. G.: Panikkar, K. R. J. Ethrophamacol. $1999,68,295$ (c) Lee, M. H.; Kim, J. Y.: Rvul. T.-H. Biol Pham Bull 2005. 28. 2253. (d) Park. E. I.: Zhao, Y. Z.; Kim, Y. C.: Sohn, D. H. Eur. J. Phamacol. 2007, 559, 115 (e) Rvul, S. Y.: Choi, S. U.; Lee, C. O.: Zee, O. P. Arch. Pham. Res. 1992, 15, 356 